\title{
MedienPädagogik
}

Zeitschrift für Theorie und Praxis der Medienbildung

\section{Zur Rekonstruktion einer Typologie jugendlichen Medienhandelns gemäß dem Leitbild der Triangulation}

\begin{abstract}
Klaus Peter Treumann, Eckhard Burkatzki, Mareike Strotmann und Claudia Wegener
Die im Folgenden dargestellten Ergebnisse sind im Rahmen des von der DFG geförderten Forschungsprojekts „Eine Untersuchung zum Mediennutzungsverhalten 12- bis 20-Jähriger und zur Entwicklung von Medienkompetenz im Jugendalter“ entstanden, das gemeinsam von Klaus Peter Treumann, Uwe Sander und Dorothee Meister geleitet wird. Das Forschungsprojekt untersucht das Medienhandeln Jugendlicher sowohl hinsichtlich Neuer als auch alter Medien. Zum einen fragen wir dabei nach den Ausprägungen von Medienkompetenz in verschiedenen Dimensionen und zum anderen konzentrieren wir uns auf die Entwicklung einer empirisch fundierten Typologie jugendlichen Medienhandelns. Methodologisch ist die Untersuchung an dem Leitbild der Triangulation orientiert und kombiniert qualitative und quantitative Zugänge zum Forschungsfeld in Form von Gruppendiskussionen, leitfadengestützten Einzelinterviews und einer Repräsentativerhebung.
\end{abstract}

\section{Fragestellung}

Das Medienhandeln Jugendlicher umfasst nach unserem Verständnis nicht nur Variablen der Mediennutzung, sondern schließt darüber hinaus solche Konzepte ein, wie sie in verschiedenen systematischen Entwürfen zur Medienkompetenz entwickelt worden sind. Als theoretische Folie nutzen wir vor allem das Bielefelder Medienkompetenz-Modell (Baacke 1996a, 1996b; Baacke u.a. 1999), um das Medienhandeln Jugendlicher zum einen facettenreich zu operationalisieren und zum anderen inhaltlich in voneinander unterscheidbare Teilbereiche zu untergliedern. Im quantitativen Teil der Studie haben wir explorativ - u.a. mit Hilfe des multivariaten Verfahrens der Clusteranalyse - vor allem die folgenden Fragen in den Mittelpunkt der Untersuchung gestellt: (a) Lassen sich die Jugendlichen in unterschiedliche Mediennutzungstypen aufteilen, die sich in ihren Interessen und medienbezogenen Umgangsformen bedeutsam voneinander unterscheiden? (b) Welche Differenzen zeigen sich bei den unterschiedlichen „Nutzungstypen“ mit Blick auf die einzelnen Dimensionen des Medienkompetenz-Modells, also der Mediennutzung, der Mediengestaltung, der 
Medienkritik sowie des Medienwissens? (c) Lassen sich zwischen der Zugehörigkeit zu den einzelnen Clustertypen und externen Variablen (individuelle Interessenlagen, soziokulturelle Orientierungen etc.), d.h solchen, die nicht bei der Clusteranalyse berücksichtigt wurden, inhaltlich plausible und statistisch signifikante Zusammenhänge finden? Im qualitativen Teil schlossen sich dann Leitfadeninterviews mit prototypischen Fällen aus den einzelnen Clustern an, die vertiefend den verschiedenen Ausprägungen des Medienhandelns sowie unterschiedlichen Formen der Medienkompetenz innerhalb eines Clusters nachgingen. Forschungsmethodologisch ging es uns gemäß dem Leitbild der Triangulation ganz besonders darum, eine möglichst enge Verknüpfung qualitativer und quantitativer Methoden zu erproben und die dazu notwendigen Verfahrensschritte bis ins Einzelne zu dokumentieren, was am Beispiel des Clustertyps der „Konsumorientierten" verdeutlicht wird.

\section{Theoretischer Bezugsrahmen}

(Medien-)Theoretische Ansätze, die für die Operationalisierung der Variablen der quantitativen Untersuchung und für die Interpretation der Ergebnisse der Clusteranalysen sowie für die qualitativen Einzelinterviews und deren Auswertung in dieser Untersuchung herangezogen worden sind, umfassen den Uses-and-Gratifications Approach, das Habituskonzept und den Kapitalsortenansatz, das Bielefelder Medienkompetenz-Modell, den Sozialökologischen Ansatz und die Wissenskluft-Hypothese. Aus Platzgründen können sie hier nicht entfaltet werden, sondern es wird auf ihre Darstellung in unserem Beitrag verwiesen, der im Jahrbuch Medienpädagogik 4 erschienen ist (Treumann u.a. 2004).

\section{Forschungsdesign und Methodologie der Studie}

\subsection{Triangulation als Leitbild}

Auch wenn heute die meisten medienpädagogischen Autoren die Notwendigkeit von Medienkompetenz betonten, existieren bislang kaum empirische Studien über Medienkompetenz bei Jugendlichen. Zudem können Differenzen zwischen einzelnen Gruppen von Jugendlichen - beispielsweise Geschlechter-, Bildungs- oder sozialräumliche Unterschiede - lediglich vermutet werden. Weiterhin ist die Entwicklung von Medienkompetenz, also ihre Genese und die Faktoren der Förderung beziehungsweise Schwächung bislang kaum untersucht worden. Die Bearbeitung eines derart komplexen Forschungsdesiderats macht es erforderlich, ein Forschungsdesign zu konzipieren, welches der inhärenten Mehrperspektivität des Untersuchungsproblems gerecht wird. Daher legen wir unsere Studie von ihrem Design her so an, dass verschiedene Erkenntnisstrategien miteinander kombiniert und aufeinander bezogen werden können. 
Ein solches Vorgehen steht in der noch relativ jungen Tradition des methodologischen Konzeptes der Triangulation (Treumann 1986, 1998; Flick 1991, 1995). Dieser Begriff wurde von Denzin $(1970,1989)$ eingeführt und stammt ursprünglich aus der Landvermessung. Er bedeutet dort eine exakte Positionsbestimmung eines Punktes von mindestens zwei unterschiedlichen anderen Punkten aus. In dieser von Denzin verwendeten Metapher spiegelt sich die Hoffnung wider, die Gültigkeit der Ergebnisse einer Studie durch die Kombination unterschiedlicher Erkenntnisstrategien - insbesondere qualitativer und quantitativer Methoden - zu verbessern (Kelle/Erzberger 1999; Treumann 1998; Engler 1997; von Saldern 1995; Wolf 1995). Dieses methodologische Prinzip scheint besonders geeignet zu sein, um die Wahrnehmungen und Deutungen von Subjekten mit den lebensweltlichen Kontexten und geronnenen sozialen Strukturen, in denen sie agieren, zu verknüpfen. Eine so verstandene Mehrperspektivität der zu bearbeitenden Forschungsfrage lässt sich auf insgesamt vier Ebenen realisieren (vgl. Treumann 1998, S. 155-157):

- Theorien-Triangulation: Eine solche Forschungsstrategie beinhaltet die Anwendung unterschiedlicher theoretischer Perspektiven und Hypothesen, um Daten zu generieren und zu interpretieren.

- Forscher-Triangulation: Diese Ebene beinhaltet die Forderung, dass mehr als eine Wissenschaftlerin oder ein Wissenschaftler dieselbe Situation untersucht, um Verzerrungen, die in der Person der Forscherin oder des Forschers liegen können, aufzudecken und zu reduzieren.

- Daten-Triangulation: In Abgrenzung zur Verwendung verschiedener Forschungsmethoden per se, die der Generierung von Daten dienen, ist mit dieser Strategie die Nutzung unterschiedlicher Datenquellen gemeint, die bei der Untersuchung sozialwissenschaftlicher Phänomene zu verschiedenen Zeitpunkten und an differierenden Orten und bei mehreren Personen - als isolierte Untersuchungseinheiten, Interaktionen praktizierend oder in sozialen Kollektiven agierend - entstehen.

- Methoden-Triangulation: Im Anschluss an Webb u.a. (1966) unterscheidet Denzin zwischen der Triangulation innerhalb einer Methode (,within-method“) und zwischen unterschiedlichen Methoden (,between-methods“). Entscheidend für die zweite Konstellation ist, dass verschiedene qualitative und quantitative Methoden der Datenerhebung und -auswertung gemäß der jeweiligen Forschungsfrage angewendet und miteinander kombiniert werden.

Die ursprüngliche Absicht, die Denzin (1970) mit der Forschungsstrategie der Triangulation intendiert hat, nämlich durch einen Mehrmethodenansatz notwendigerweise zu verlässlicheren und gültigeren Ergebnissen als bei Anwendung einer einzigen Forschungsmethode zu kommen - „Integrationsthese“ (Treumann 1998) bzw. „Konvergenzmodell“ (Kelle/Erzberger 1999) - steht inzwischen in Konkurrenz zu einer methodologischen Position, die besagt, dass die Methoden-Triangulation aufgrund der unterschiedlichen Theorietraditionen, aus denen qualitative und quantitative Methoden entstammen, breitere, vielfältigere 
und tiefere Erkenntnisse über die untersuchten sozialen Phänomene zu liefern imstande ist („Komplementaritätsthese“, Treumann 1998).

\subsection{Design}

Unsere Studie muss nicht beim „Stande Null“ beginnen, sondern kann auf einen relativ breit gefächerten Kanon entwickelter Konzepte und Theorien der Medienforschung zurückgreifen, insbesondere auf das Bielefelder Medienkompetenz-Modell (Baacke 1996a, b). Um die Fragestellungen und Zielsetzungen des Projektes bearbeiten zu können, ist es daher erkenntnistheoretisch möglich und gerechtfertigt, das „klassische“ Phasenmodell der Methodenkombination (zuerst qualitative Verfahren zur Hypothesenkonstruktion und danach quantitative Methoden zur Hypothesenüberprüfung einzusetzen) aus forschungsmethodologischen Gründen umzukehren. Daher wurden ausgewählte Konzepte der Medienkompetenz, des Nutzenansatzes und der Wissenskluft-Perspektive bzw. -Hypothese mit Hilfe von Items eines standardisierten Fragebogens operationalisiert. Daneben wurden beispielsweise Freizeitaktivitäten von Jugendlichen im Allgemeinen, ihre Zugehörigkeit zu Vereinen, Gruppen, Organisationen und jugendkulturellen Szenen, ihre Genre-Präferenzen im Hinblick auf Sendungen und Spielfilme im Fernsehen, ihre Vorgehensweisen beim Erlernen der Bedienung von Mediengeräten und zur Lösung bei der Arbeit mit dem Computer („Coping-Strategien“), ihre medial vermittelten Vorbilder und Idole sowie soziostrukturelle Variablen erhoben. Der Erhebungsplan für die standardisierte Befragung der Jugendlichen war als Querschnittsstudie angelegt.

\subsection{Stichprobe}

Die Grundgesamtheit setzte sich aus Jugendlichen der drei Bundesländer Mecklenburg-Vorpommern, Nordrhein-Westfalen und Sachsen-Anhalt zusammen, die zum Befragungszeitraum ein Lebensalter von 12 bis einschließlich 20 Jahren erreicht hatten, Deutsch als Muttersprache beherrschten ${ }^{1}$, mindestens drei Jahre in demselben Ort und jeweils in einem der drei folgenden Sozialräume wohnten: (a) Ländliche Wohngegenden (<25.000 Einw.), (b) Mittelstädte (<50.000 Einw.) und (c) Großstädte (> 100.000 Einw.): Aus der Grundgesamtheit wurde zum einen eine zweifach geschichtete Flächenstichprobe gezogen, die sich zum einen auf die drei Bundesländer und zum anderen auf die drei Sozialräume bezog. In einem letzten Schritt wurde in den drei ausgewählten Sozialräumen per Zufall mindestens je eine Hauptschule, Realschule beziehungsweise Sekundarschule, ein Gymnasium, eine Gesamtschule (für NRW)

1 Das Medienhandeln von Jugendlichen aus türkischen Migrantenfamilien wird in einem gesonderten Dissertationsvorhaben von Mareike Strotmann analysiert, das den Arbeitstitel „Mediennutzung im Alltag türkischer Jugendlicher“ trägt. 
und eine Berufsschule ausgewählt und die Jugendlichen aus mindestens einer Schulklasse pro Schülerjahrgang von der siebenten Jahrgangsstufe oder dem ersten Ausbildungsjahr an aufwärts befragt. Es ergab sich über alle Bundesländer hinweg eine bereinigte Nettostichprobe von insgesamt $n=3.271$ Jugendlichen. Diese Stichprobe bildete den Ausgangspunkt für sämtliche nachfolgenden Untersuchungen.

\subsection{Methoden der Datenerhebung}

\subsubsection{Standardisierte Befragung}

Die Umfrage zum Mediennutzungsverhalten und zur Medienkompetenz Jugendlicher erfolgte mit Hilfe eines standardisierten Fragebogens, der insgesamt 121 Fragen (-komplexe) umfasste (siehe auch Abschnitt 3.2) und zuvor einem Pretest unterzogen wurde. Der Erhebungszeitraum erstreckte sich von Mitte Oktober bis Anfang Dezember 2001.

\subsubsection{Leitfadengestützte qualitative Einzelinterviews}

Aus den sieben Clustern der Clusteranalyse (siehe Abschnitt 4.1.2) wurden jeweils diejenigen fünf Jugendlichen ausgewählt, die einen möglichst geringen Abstand zum Clustercentroid beziehungsweise -Schwerpunkt aufwiesen. Insgesamt wurden daher 7 x 5 = 35 Jugendliche mit Hilfe eines Leitfadens qualitativ interviewt. ${ }^{2}$ Zentrale inhaltliche Themenschwerpunkte sind in Abschnitt 4.3.1 aufgeführt. Die qualitativen Interviews mit den ausgewählten Jugendlichen wurden in allen drei Bundesländern von Juni bis Juli 2002 geführt.

\subsection{Methoden der Datenanalyse}

Den Ausgangspunkt der nachfolgenden multivariaten Analysen bildeten $\mathrm{m}=141$ Variablen (z.B. Fragebogenitems/Schätzskalen), mit Hilfe derer acht der neun Unterdimensionen des Bielefelder Medienkompetenz-Modells operationalisiert wurden (nicht berücksichtigt wurde die innovative Mediengestaltung, da uns die valide Erfassung bzw. Messung von Veränderungen und zur Weiterentwicklung des Mediensystems mit Hilfe von standardisierten Fragebogenitems nicht adäquat umsetzbar erschien).

2 In Abweichung von der allgemeinen Vorgabe konnten im Clustertyp der „Konsumorientierten“ sechs Fälle leitfadengestützt interviewt werden. 


\subsubsection{Hauptkomponentenanalysen}

Für jede der acht Unterdimensionen des Medienkompetenz-Modell wurde zwischen den entsprechenden Variablen eine Korrelationsmatrix errechnet und mit Hilfe der Hauptkomponentenanalyse (vgl. Backhaus u.a. 1996; Bortz 1999; Tabachnik/Fidell 1996) eine Reihe von Komponenten extrahiert. Die Bestimmung der Anzahl der zu extrahierenden Hauptkomponenten erfolgte durch eine kombinierte Anwendung des Kaiser-GuttmanKriteriums, des Scree-Tests nach Catell sowie aufgrund inhaltlicher Gesichtspunkte. Danach wurden die Komponenten zu ihrer besseren Interpretierbarkeit gemäß dem Varimax-Kriterium auf eine orthogonale Einfachstruktur hin rotiert. In einem weiteren Auswertungsschritt wurden die Hauptkomponentenwerte für jeden der befragten Jugendlichen auf den 32 Hauptkomponenten berechnet. Sie sind als z-Werte skaliert und lassen sich als Ausprägungen interpretieren, welche die Jugendlichen auf den einzelnen Hauptkomponenten erreichen. Diese varianzstarken bzw. erklärungsmächtigen Variablenbündel, die auf einer höheren Abstraktionsstufe als die Eingangsvariablen liegen und damit einen größeren Verallgemeinerungsgrad besitzen, bilden die Ausgangsdaten für die von uns durchgeführten Clusteranalysen. Über die Ergebnisse der Hauptkomponentenanalysen und die sich aus ihnen ergebenden Konsequenzen für die Rekonstruktion der Binnenstruktur der vier Dimensionen des Bielefelder Medienkompetenzmodells ist bereits an anderer Stelle berichtet worden (siehe Treumann u.a. 2004). Die 32 erhaltenen Komponenten bilden die Abszisse des Profildiagramms der Clustertypen (siehe Abbildung 2).

\subsubsection{Clusteranalysen}

Das Ziel der vorliegenden Arbeit, nämlich die Entwicklung einer empirisch gestützten Typologie des Medienhandelns Jugendlicher, deren theoretische Fundierung vor allem im Bielefelder Medienkompetenz-Modell liegt, wird mit Hilfe des multivariaten Verfahrens der Clusteranalyse (vgl. Arabie/Hubert/de Soete 1996; Bacher 1996, 2002; Bailey 1994; Eckes/Rossbach 1980; Everitt 1993; Gordon 1999) zu erreichen versucht. Folgende Hypothese wurde zugrunde gelegt. Es lassen sich in Bezug auf ihre Medienkompetenz inhaltlich voneinander unterscheidbare Gruppen von Jugendlichen rekonstruieren. Der Zweck der hier applizierten Clusteranalysen ist es, unterschiedliche Gruppen von Jugendlichen aufgrund ihrer Ausprägungen auf den Hauptkomponenten der verschiedenen Unterdimensionen der Medienkompetenz derart zu klassifizieren, dass die Heranwachsenden innerhalb einer Gruppe bzw. eines Clusters sehr wenig differieren (Prinzip der Homogenität) und Jugendliche aus unterschiedlichen Gruppen sich möglichst stark voreinander unterscheiden (Prinzip der Heterogenität). Die Klassifikation der 12- bis 20-Jährigen auf der Basis ihrer Medienkompetenz erfolgte in mehreren Schritten: 
Zuerst wurden aus dem Datensatz diejenigen Jugendlichen ausgeschieden, die auf den 32 Hauptkomponenten mehr als zehn fehlende Werte (Missing Values) aufwiesen ${ }^{3}$ Es ergab sich eine Analysestichprobe von insgesamt $n=1.662$ Heranwachsenden. Danach ist als Proximitätsmaß zwischen den Personenpaaren der Stichprobe - bezogen auf die Hauptkomponenten - die quadrierte Euklidische Distanz berechnet worden. Hierarchische Clusteranalysen nach Ward wurden deswegen durchgeführt, um die Anzahl der Personencluster und die Anfangsschätzungen für die Clusterschwerpunkte zu bestimmen. Aufgrund der Ergebnisse des inversen Scree-Tests und gemäß dem Kriterium einer möglichst großen inhaltlichen Prägnanz entschieden wir uns für eine Sieben-Cluster-Lösung. Da die hierarchischen Clusteranalyseverfahren den Nachteil besitzen, irreversibel zu sein, d.h. also auch bei Vorliegen weiterer Informationen keine neuen Zuordnungen der Personen zu einem anderen Cluster zu ermöglichen, wenn die Entscheidung über die Zugehörigkeit zu einem Cluster erst einmal gefallen ist, wurde in einem abschließenden Schritt die Clusterzentrenanalyse (K-means-Verfahren) angewendet. Ausgehend von der über das Wardsche Verfahren ermittelten Clusteranzahl sind die zu clusternden Personen mit Hilfe eines Austauschalgorithmus so lange hin- und hergeordnet worden, bis eine Minimierung der Distanz zwischen der jeweiligen Person und dem Clusterzentroid und damit eine maximale Homogenität innerhalb des entsprechenden Clusters erreicht wurde.

\section{Ergebnisse}

Da in dieser Veröffentlichung der methodologische Ansatz der Methodentriangulation den Schwerpunkt bildet, führen wir inhaltliche Ergebnisse vor allem zur Illustration der von uns gewählten Auswertungsstrategie an. Sie sind damit - schon allein aus Platzgründen - sehr selektiv. Eine systematische und differenzierte Darstellung der Einzelergebnisse wird in einer Buchpublikation erfolgen, die im Laufe des Jahres 2004 erscheinen wird.

\subsection{Ergebnisse der Clusteranalysen}

Im Rahmen der quantitativen Analysen lässt sich eine aus insgesamt sieben Cluster bestehende Typologie rekonstruieren, die das Medienhandeln Jugendlicher angemessen und prägnant beschreibt. Bevor im Folgenden mit den „Konsumorientierten“ einer der von uns ermittelten Cluster ausführlicher dargestellt wird, soll der Blick zunächst auf die empirische Verteilung der Clusterzugehö-

3 Wir folgen einer Auswertungsheuristik, die Bacher (2002; 1996) für den Umgang mit fehlenden Werten bei der Anwendung von Clusteranalysen empfiehlt. 


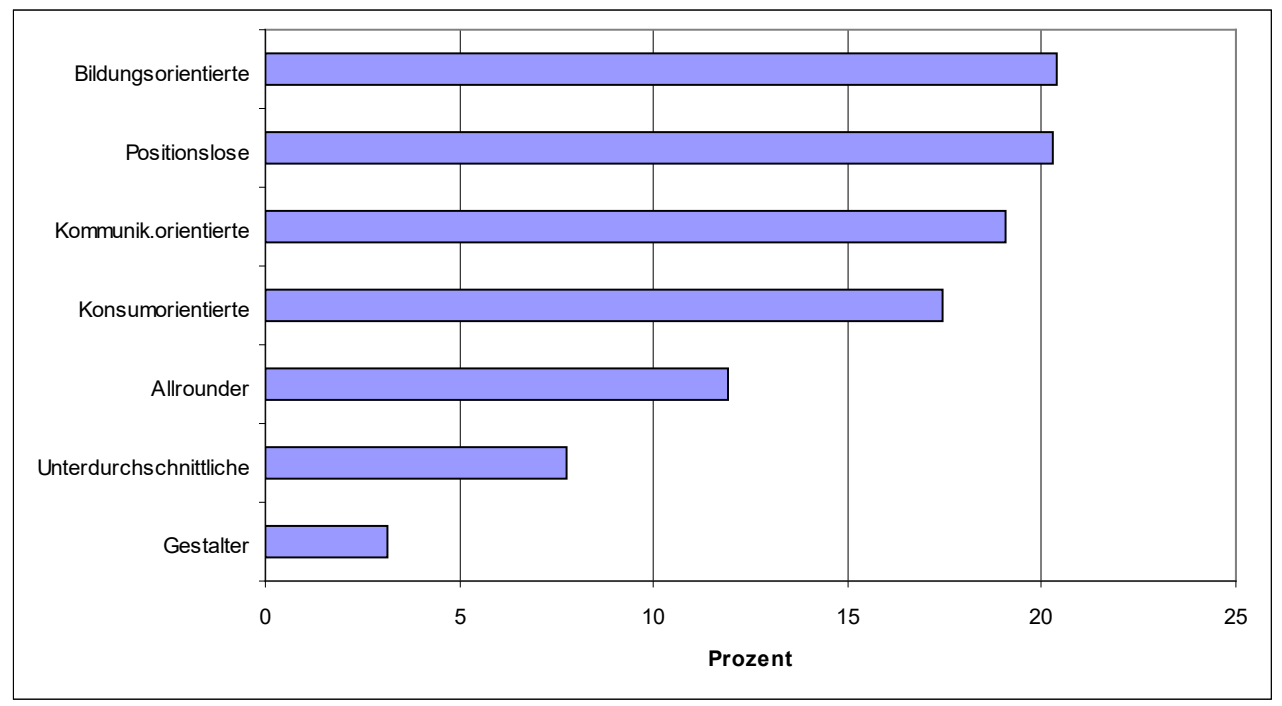

Abb. 1: Zur Verteilung der Clusterzugehörigkeit in einer Auswahlstichprobe von Jugendlichen zwischen 12 und 20 Jahren $(n=1662)$. Ausgeschlossen aus den Analysen wurden die Fälle, die auf den 32 Hauptkomponenten jugendlichen Medienhandelns mehr als zehn fehlende Werte aufwiesen.

rigkeiten in unserer Untersuchungsstichprobe gerichtet werden, um anschließend kurz auf die einzelnen Clustertypen einzugehen.

Bezogen auf die Auswahlstichprobe der in die Clusteranalyse einbezogenen Fälle lässt sich an dieser Stelle zunächst konstatieren, dass die Anteile der auf die verschiedenen Cluster entfallenden Jugendlichen in ihrer Höhe variieren (vgl. Abbildung 1). Den jeweils größten Anteil stellen mit 20,4 bzw. 20,3 Prozent die Bildungsorientierten und die Positionslosen unter den von uns befragten Jugendlichen dar. Die Bildungsorientierten zeichnen sich im Kollektiv der jugendlichen Mediennutzer insbesondere durch eine weit überdurchschnittliche Nutzung von Belletristik und Sachliteratur sowie von Zeitungen aus. Typisch und prägnant für die Bildungsorientierten ist ferner, dass sie die Gruppe mit dem mit Abstand fundiertesten literarischen Bildungswissen sind. Schaut man sich die soziodemographische Verteilung innerhalb des Clusters an, wird deutlich, dass es insbesondere die höher gebildeten Mädchen sind, die den Bildungsorientierten zuzuordnen sind. Die Präferenz für Bücher scheint demnach nach wie vor geschlechtsspezifisch ausgeprägt zu sein. So konnte die Studie „Medienwelten Jugendlicher“ bereits Ende der 80er Jahre nachweisen, dass Bücher deutlich höher in der Gunst der Mädchen als in der der Jungen stehen (vgl. Baacke/Sander/Vollbrecht 1990).

Die auf dem zweiten Rang stehenden Positionslosen stellen geradezu ein Kontrastpaar zu den Bildungsorientierten dar. Dem stark überdurchschnittlichen Gebrauch von Printmedien und der unterdurchschnittlichen Nutzung der audiovisuellen Medien bei den Bildungsorientierten steht die deutlich unterdurchschnittliche Printmediennutzung und die überdurchschnittliche Nutzung 
audiovisueller Medien der Positionslosen gegenüber. Ähnliche Gegenläufigkeiten finden sich auch im Bereich informativen Medienkunde: So sind die Positionslosen hier mit vergleichsweise sehr geringen Kenntnissen ausgestattet. Ihren Namen verdanken die Positionslosen aber ihren durchgängig extrem unterdurchschnittlichen Kennwerten im Bereich der medienkritischen Einstellungen. Nicht völlig ausgeschlossen ist, dass sich gerade in der unkritischen Haltung ein Alterseffekt widerspiegelt; sind es doch eher die jüngeren und zudem männlichen sowie schließlich unterdurchschnittlich Gebildeten, die dieses Cluster vornehmlich repräsentieren.

Deutlich mehr als 15 Prozent unter den Jugendlichen machen die Kommunikationsorientierten aus. Das Profil ihres Medienhandelns ist in Abbildung 2 darstellt. ${ }^{4}$ Sie bilden einen kontrastierenden Gegentypus zu dem weiter unten ausführlicher beschriebenen Cluster der Konsumorientierten. Die Kommunikationsorientierten - eher die weiblichen Jugendlichen jüngeren Alters - zeichnen sich durch ein stark überdurchschnittliches Nutzungsinteresse an Musik, Infotainment und Unterhaltung aus, verbunden mit einer stark ausgeprägten kommunikativen Orientierung, die sich insbesondere im Umgang mit Neuen Medien zeigt. Sowohl auf dem Gebiet des literarischen Bildungswissens als auch im Bereich des Wissens über das Mediensystem weist dieses Cluster unter-

4 Während, auf der Abszisse die 32 Hauptkomponenten als empirisch rekonstruierte Teilaspekte der vier Dimensionen des Bielefelder Medienkompetenz-Modells platziert wurden, sind auf der Ordinate die z-Werte abgetragen. Die Profillinie für ein bestimmtes Cluster ergibt sich aus der Verbindung der Koordinaten seines Centroids bzw. Schwerpunkts im 32-dimensionalen Raum, der durch alle Hauptkomponenten aufgespannt wird. Beispielsweise ergibt sich ein z-Wert von rund 0,9 für das Cluster der Konsumorientierten auf der Hauptkomponente „Kopieren und Duplizieren“ als einem Teilaspekt der rezeptiven Mediennutzung dadurch, dass die z-Werte aller Angehörigen des Clusters der Konsumorientierten, die ihre individuellen Ausprägungen auf dieser Komponente darstellen, gemittelt werden, d.h. in unserem Fall aufaddiert und durch $\mathrm{n}=290$ dividiert werden. Alle z-Werte mit einem positiven Vorzeichen sind größer und alle z-Werte mit einem negativen Vorzeichen sind kleiner als der Mittelwert der z-Skala, der gleich Null ist und dem Durchschnitt der Gesamtstichprobe der Jugendlichen entspricht. Der sich in unserem Beispiel als arithmetischer Mittelwert von $\mathrm{z}=+0,9$ ergebende Profilpunkt für die Hauptkomponente „Kopieren und Duplizieren“ bedeutet, dass die Konsumorientierten im Vergleich zur Gesamtstichprobe aller Jugendlichen - sie wird durch den Wert z $=0$ symbolisiert - dieser Medienaktivität in weit überdurchschnittlichem Ausmaß nachgehen. Ein negativer z-Wert, wie etwa -0,75 für das „selbstgesteuert-systematische Lösungsverhalten“ bei Problemen im Umgang mit Medien, signalisiert, dass ein solches Verhalten von den Konsumorientierten vergleichsweise sehr selten praktiziert wird. Unter der Voraussetzung, dass die Hauptkomponentenwerte für die einzelnen Komponenten der Stichprobe aus normalverteilten Grundgesamtheiten stammen, lassen sich in erster Näherung die z-Werte mit Hilfe von Prozenträngen, die ihnen jeweils zugeordnet werden können, anschaulich interpretieren. Der Prozentrang gibt an, wie groß der Anteil von Personen ist, deren Werte unterhalb einer bestimmten Merkmalsausprägung (z.B. eines z-Wertes) liegen. So entspricht im obigen Beispiel ein z-Wert von + 0,9 einem Prozentrang von 81,6\% (siehe z.B. Abel/Möller/Treumann 1998, Tabelle A, S. 190-191), d.h. ein Jugendlicher der dem Clustertyp der Konsumorientierten angehört und ganz in der Nähe des Clusterzentroids läge, überträfe mit seiner Ausprägung auf der Hauptkomponente „Kopieren und Duplizieren“ 81,6\% der Jugendlichen der Gesamtstichprobe. Oder anders ausgedrückt: Nur 19,4\% (= 100\% - 81,6\%) aller Jugendlichen würden - im Vergleich zu einem Jugendlichen, der im Schwerpunkt des Clusters läge - extremere Ausprägungen auf dieser Hauptkomponente aufweisen. 


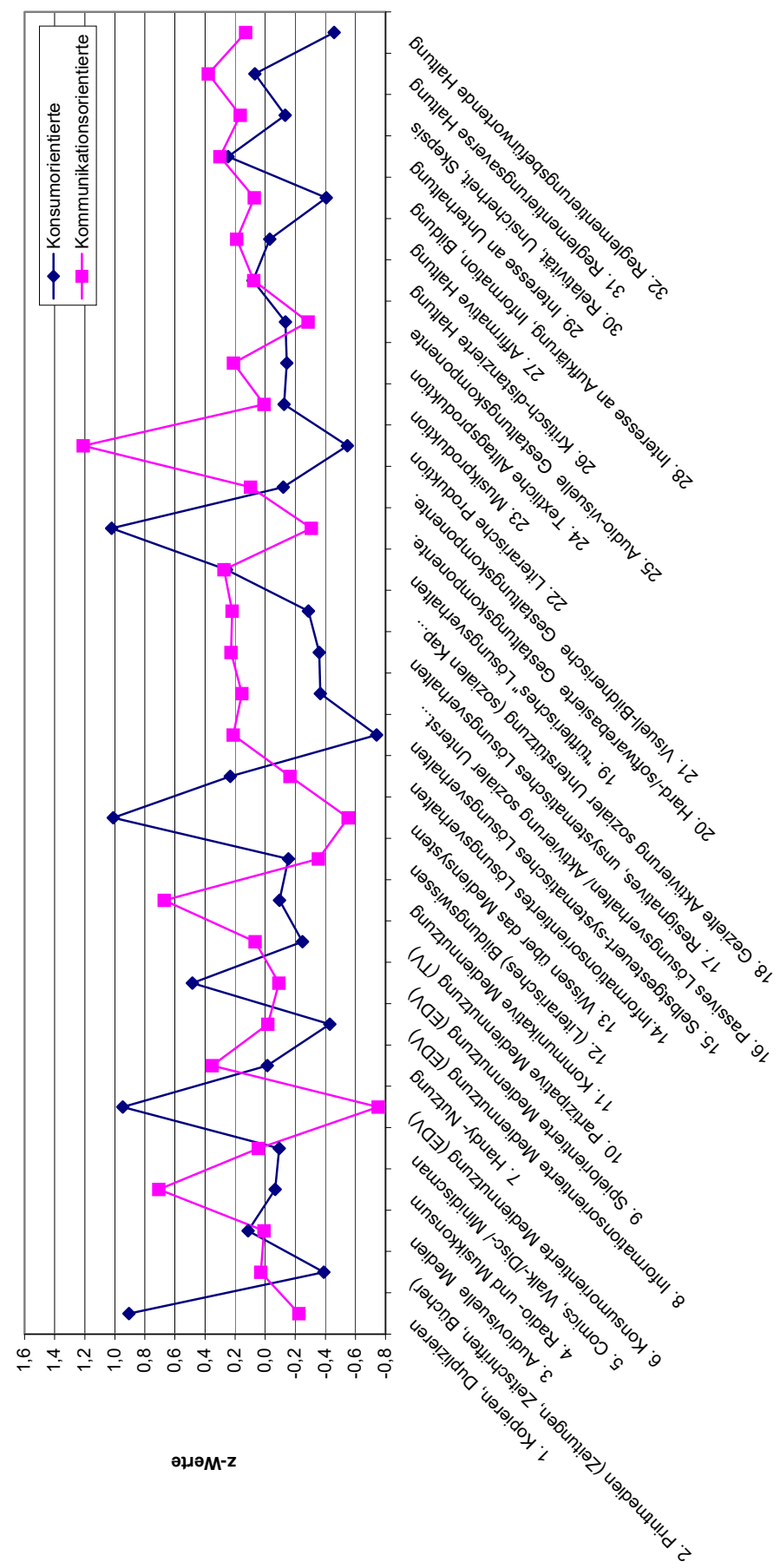

Abb. 2: Profildiagramm des Clustertypen der „Konsumorientierten“ und „Kommunikationsorientierten“ entlang der 32 Hauptkomponenten jugendlichen Medienhandelns. Die Kommunikationsorientierten werden in dieser Abbildung lediglich zu Illustrationszwecken als Gegentypus zu den Konsumorientierten dargestellt. Eine ausführliche Darstellung dieses Clusters bleibt einer späteren Veröffentlichung Vorbehalten. 
durchschnittliche Kenntnisse auf. Anstatt sich Wissensvorräte langfristig umfänglich anzueignen, erschließen sich die Kommunikationsorientierten dieses Wissen möglicherweise je nach Bedarf über soziale Kontakte oder Netzwerke.

Ein weiteres kontrastierendes Paar hinsichtlich der Struktur ihres Medienhandelns stellen die Allrounder und Unterdurchschnittlichen dar, die für sich gesehen jeweils 11,9 und 7,8 Prozent der Gesamtstichprobe umfassen. Kennzeichnend für die sog. Allrounder ist unseren Analysen zufolge, dass sie - verglichen mit der Nutzungsintensität anderer Jugendlicher - sowohl von alten als auch von den Neuen Medien mit stark überdurchschnittlicher Häufigkeit Gebrauch machen. Bei den Angehörigen dieses Clusters beschränkt sich der Umgang mit Neuen Medien allerdings nicht auf deren anwendungsbezogene Nutzung. Typisch und prägnant für die Allrounder ist vielmehr, dass sie sich ebenfalls gestalterisch an ihren Medien betätigen. In übergreifender Betrachtung stellen sie unter den Jugendlichen diejenige Gruppe dar, die intensiver und zugleich selbstbestimmter als andere die Klaviatur der Medien für ihre eigenen Zwecke nutzen können. Erwartungsgemäß weisen sie ein überdurchschnittliches Wissen über die Sachzusammenhänge des Mediensystems auf. Unter soziodemografischen Gesichtspunkten handelt es sich bei den Allroundern um ein stark männlich dominiertes Cluster von Jugendlichen jüngeren und mittleren Alters (12 bis 17 Jahre), in dem insbesondere die Gruppe der Realschüler stark überrepräsentiert ist.

Im Gegensatz zu den Allroundern handelt es sich bei den Unterdurchschnittlichen um ein Cluster, das als Typus jugendlichen Medienhandelns - bezogen auf die verschiedenen Dimensionen des Bielefelder Medienkompetenzmodells - vorwiegend defizitär bestimmt ist. So weisen die Angehörigen dieses Clusters im Vergleich zur Gesamtstichprobe fast durchgängig in allen Bereichen der Mediennutzung - mit Ausnahme der Rezeption audiovisueller Medien (Fernsehen, Video, Kino) - unterdurchschnittliche Kennwerte der Mediennutzung auf Dies gilt sowohl für den Bereich der rezeptiven als auch für den der interaktiven Mediennutzung. Auch gestalterisch treten die Unterdurchschnittlichen im Umgang mit den ihnen verfügbaren Medien mit einem vergleichsweise geringen Engagement in Erscheinung. Sie haben ein nur sehr geringes Wissen über die Gegebenheiten des Mediensystems und weisen entsprechend im Umgang mit Problemen, die im Kontext des Mediengebrauchs auftreten, kein aktiv strukturiertes Handlungsprofil auf. Auf der Ebene ihrer medienkritischen Einstellungen fallen die Angehörigen dieses Clusters im Weiteren durch ein relativ hohes Vertrauen in den Realitätsgehalt medialer Wirklichkeitsdarstellungen auf. Unter soziodemografischen Gesichtspunkten handelt es bei den Unterdurchschnittlichen um ein Cluster vorwiegend männlicher Jugendlicher jüngeren Alters aus dem unteren und mittleren Bildungssegment (Hauptschulen, Sekundarschulen, Realschulen).

Eine deutliche Minderheit innerhalb der Untersuchungspopulation stellen schließlich die Gestalter dar. Unter 32 Jugendlichen findet sich im Durchschnitt nur ein einziger, der mit den audiovisuellen Medien gestalterisch um- 
geht. Entsprechend verdanken die Gestalter, bei denen es sich überraschenderweise eher um die jüngeren, niedriger gebildeten Jugendlichen handelt, ihren Namen ihren ausgeprägt gestalterischen Ambitionen im Umgang mit Medien. Diese Zuschreibung gilt in herausragender Weise für den kreativen Umgang mit audiovisuellen Medien in der Freizeit (z.B. Radiosendungen moderieren, Hörspiele gestalten, Filme drehen), aber auch für den Bereich der literarischen Produktion. Die Gestalter sind damit das einzige Cluster, das auf beinahe allen Ebenen der kreativen Mediengestaltung überdurchschnittliche Werte vorweisen kann. Anders, als man es in diesem Cluster möglicherweise erwarten würde, verfügen Gestalter auf dem Gebiet der informativen Medienkunde jedoch über keinen überdurchschnittlichen Kenntnisreichtum. Ihr literarisches Bildungswissen ist knapp unterdurchschnittlich, ihr Wissen über das Mediensystem bewegt sich auf einem ähnlichen Niveau.

\section{Konsumorientierte}

Auf das Cluster der Konsumorientierten entfallen etwa 17 Prozent der Jugendlichen aus der für die Clusteranalysen herangezogenen Auswahlstichprobe. Das Profil des Medienhandelns für diesen Clustertyp ist in Abbildung 2 dargestellt.

Die Konsumorientierten zeichnen sich im Kollektiv jugendlicher Mediennutzer dadurch aus, dass ihr Mediengebrauch in erster Linie Zwecken des Konsums und der Unterhaltung dient. Sie zeigen in diesem Zusammenhang - ähnlich wie die Allrounder eine sehr starke Zuwendung zu dem Bereich der Neuen Medien. Ihr Nutzungsstil dieser Medien ist dabei aber nur wenig durch gestalterische Aktivitäten geprägt.

In dem Bereich der rezeptiven Mediennutzung zeigen Konsumorientierte einen extrem überdurchschnittlichen Grad der Beschäftigung mit dem Kopieren und Duplizieren medialer Inhalte und sind hier noch etwas aktiver als die Gruppe der Allrounder. Parallel hierzu zeigt sich ein stark unterdurchschnittlicher Nutzungsgrad des Printmediensektors, und zwar sowohl von Romanen und Sachbüchern als auch - mit leicht mäßigerer Ausprägung - von Zeitungen und Zeitschriften. Die Nutzungsintensität audiovisueller Medien wie Fernsehen, Video und Kino fällt leicht überdurchschnittlich, die von Radio und Musikkassetten eher unterdurchschnittlich aus. Analog hierzu findet auch der Gebrauch des Walk- oder Discmans in dieser Gruppe eine leicht unterdurchschnittliche Resonanz.

Neuen Medien gegenüber sind die Konsumorientierten, wie bereits angedeutet, sehr aufgeschlossen. Dies zeigt sich bei den Angehörigen dieses Clusters sowohl an der extrem überdurchschnittlichen Nutzungsintensität von Computer und Internet als auch an dem immerhin durchschnittlichen Nutzungsgrad des Handys. In der Art der Nutzung dieser Medien spiegelt sich aber ihr überragendes Konsum- und Unterhaltungsinteresse wider. So weisen sie einerseits einen extrem überdurchschnittlichen Kennwert auf der Ebene der konsumorientierten EDV-Nutzung (Downloading von Software, Herunterladen und Anhören von Musikdateien, Abwicklung von Kaufgeschäften über das Internet etc.) auf. 
Zum anderen neigen sie in stark erhöhtem Maße zur spielorientierten Nutzung von Computer und Internet. Stark unterdurchschnittlich fällt hier hingegen das Ausmaß der informationsorientierten Mediennutzung aus. Gleiches gilt im Weiteren für alle Formen der partizipativen Mediennutzung, die in der von uns gewählten Operationalisierung ein Nachfragen nach Informationen bei öffentlichen und privaten Sendeanstalten mit einschließt.

Gestalterisch beschränkt sich der Aktivitätsradius der Konsumorientierten auf hardund softwarebasierte Gestaltungsaktivitäten, deren Häufigkeit bei ihnen im Vergleich zu den anderen Clustern allerdings extrem überdurchschnittlich ausgeprägt ist. Abgesehen von ihrem tüftlerischen Engagement am EDV-Gerät weisen bei ihnen die Aktivitätswerte in dem Bereich der kreativen Mediengestaltung mittel bis stark unterdurchschnittliche Werte auf. Dies gilt für den visuell-bildnerischen und den audio-visuellen Bereich, für die literarische und textliche Alltagsproduktion wie auch für den Bereich musikalischer Aktivitäten.

Prägnant ist im Weiteren die Art des bevorzugten Lösungsverhaltens, das Konsumorientierte im Umgang mit technischen Problemen im Kontext der Mediennutzung zeigen. So haben sie einerseits eine leicht überdurchschnittliche Präferenz für informationsorientierte und tüftlerische Lösungsansätze. Hiermit einher geht prekärerweise aber eine extrem unterdurchschnittliche Häufigkeit in der Anwendung selbstgesteuert-systematischer Lösungsstrategien. Sehr unterdurchschnittlich fällt im Weiteren auch die Häufigkeit für solche Strategien aus, die für Zwecke der Problemlösung auf die mehr oder weniger gezielte Aktivierung sozialer Unterstützung setzen. Betrachtet man den Bereich der informativen Medienkunde, so treten Konsumorientierte auch hier mit einem eigenen Kompetenzprofil in Erscheinung. Ein eher durchschnittliches literarisches Bildungswissen kombiniert sich auf diesem Gebiet mit einem extrem überdurchschnittlich ausgeprägten Wissen über das Mediensystem.

In dem Bereich der medienkritischen Einstellungen weisen Konsumorientierte ebenfalls ein typisches Einstellungsmuster auf. Auf der Ebene der analytischen Medienkritik zeigen sie sich gegenüber der Wirklichkeitsdarstellung in den Medien in leicht überdurchschnittlichem Maße kritisch-distanziert. Mit anderen Worten: Sie sind sich implizit bewusst, dass Realitätsdarstellungen in den Medien strukturellen Verzerrungen unterliegen. Dabei ist jedoch gleichzeitig ein durchschnittliches Grundvertrauen in die Mediendarstellung gegeben.

Im Weiteren haben Konsumorientierte, so zeigen die Analysen zur reflexiven Medienkritik, im Zuge der Rezeption medialer Inhalte ein stark unterdurchschnittliches Interesse an Bildung und Aufklärung. Stark überdurchschnittlich ist demgegenüber, wie oben bereits angedeutet, ihr Interesse an Unterhaltung und Infotainment. Mit Blick auf die Frage, wie weit sie richtige von falschen Mediendarstellungen unterscheiden können, sind Skepsis und Unsicherheit leicht unterdurchschnittlich ausgeprägt.

Hinsichtlich der ethischen Kritikdimension fällt auf, dass Konsumorientierte Reglementierungsversuchen im Hinblick darauf, was in den Medien gezeigt werden darf, extrem kritisch gegenüber stehen. Aversionen gegenüber Regle- 
ments sind entsprechend - verglichen etwa mit den Bildungsorientierten - relativ stark ausgeprägt, wenngleich sie bei den Angehörigen dieses Clusters nur ein insgesamt durchschnittliches Niveau erreichen.

\subsection{Bivariate Zusammenhänge zwischen der Zugehörigkeit zum Clustertypus der „Konsumorientierten “ und ausgewählten externen Variablen}

Die vorliegende Untersuchung bleibt aber nicht bei der Entwicklung einer empirisch fundierten Typologie des Medienhandelns Jugendlicher stehen, sondern evaluiert in einem weiteren Schritt die gewonnene clusteranalytische Lösung, indem sie danach fragt, ob sich zwischen der Zugehörigkeit zu einem bestimmten Cluster und einer Reihe von externen Variablen inhaltlich plausible und statistisch signifikante Zusammenhänge ergeben. Externe Merkmale sind solche, die nicht in die clusteranalytisch Typenbildung eingegangen sind. Aus Platzgründen beschränken wir uns zum einen auf das Cluster der Konsumorientierten und zum anderen auf eine Teilmenge der externen Variablen. Da die Konsumorientierten die Neuen Medien intensiv nutzen (z.B. zum Kopieren und Duplizieren), gehen wir aufgrund mediensozialisatorischer Überlegungen davon aus, dass in diesem Cluster ein signifikant höherer Anteil Jungenanteil und komplementär dazu ein geringerer Prozentsatz von Mädchen zu erwarten ist. ${ }^{5}$ Wenn eine derartige Vorhersage mit den empirisch erhobenen Daten zur Geschlechterverteilung kompatibel ist, dann darf ein solcher Befund als ein Beleg (neben anderen) für die Gültigkeit dieses Clustertyps angesehen werden. In diesem Beitrag wird über die Enge bzw. Stärke der Zusammenhänge zwischen der Zugehörigkeit zum Cluster der Konsumorientierten und den externen Variablen der Geschlechterrolle der Jugendlichen, ihren individuellen Interessenlagen (hier dem Technikinteresse), ihren informellen Zugehörigkeiten oder Selbstzuschreibungen zu jugendkulturellen Szenen (von Computerfreaks über Umweltschützer bis hin zu Menschenrechtlern), den situativen und sozialen Kontexte ihrer Computer- und Internetznutzung sowie über das Ausmaß ihrer Praktizierung von nichtmedienbezogenen Freizeitaktivitäten berichtet.

Ähnlich wie bei den Neuen Medien gegenüber ebenfalls aufgeschlossenen Allroundern handelt es sich auch bei den Konsumorientierten um ein männlich dominiertes Cluster (vgl. Abbildung 3). 90 Prozent aller konsumorientierten Jugendlichen in unserer Untersuchungsstichprobe sind männlich, nur etwa 10 Prozent weiblich. Hiermit in Einklang steht - geschlechtsspezifische Rol-

5 Wir prüfen also anhand des Assoziationsmaßes Cramers V und der sich ergebenden Prozentanteile in den betreffenden Merkmalsausprägungen, ob sich ein solcher statistisch bedeutsamer und inhaltlich substanzieller Zusammenhang zwischen dem Cluster der Konsumorientierten (zugehörig versus nicht zugehörig) und dem Geschlecht (männlich oder weiblich) ergibt. Zur Verdeutlichung und Kontrastierung sind in den Abbildungen ebenfalls die prozentualen Häufigkeiten der entsprechenden Merkmalsausprägungen in der Gesamtstichprobe angegeben. 


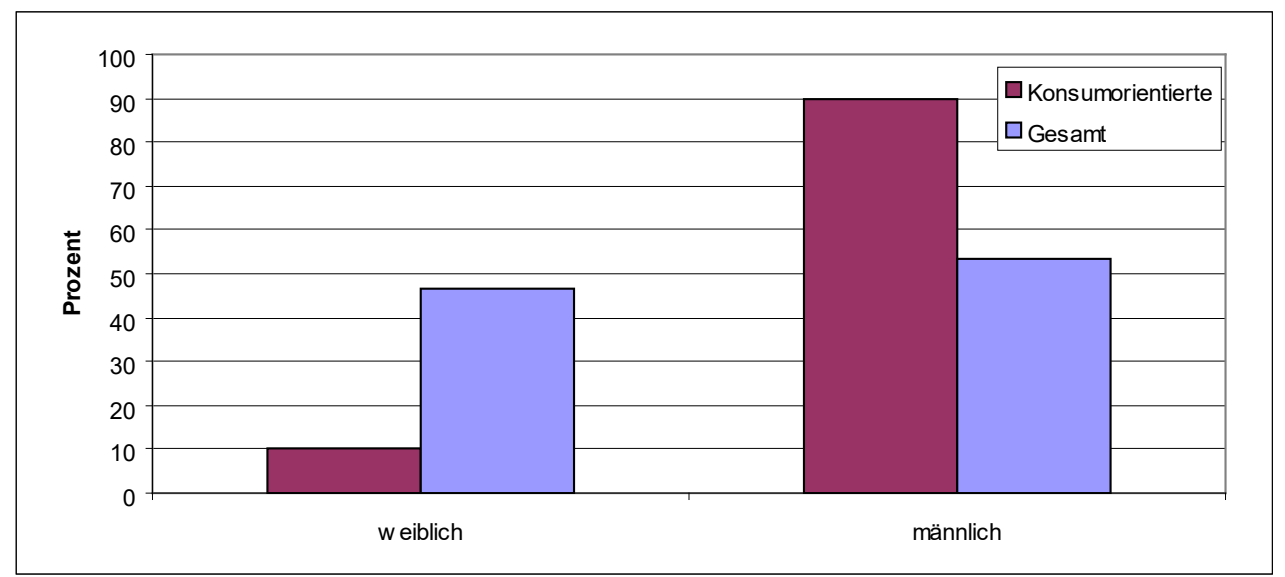

Abb. 3: Prozentualer Anteil von Jungen und Mädchen im Cluster der Konsumorientierten und in der Gesamtstichprobe (n [Gesamt] = 1662; $n$ [Konsumorientierte] = 290). Ausgeschlossen aus den Analysen wurden die Fälle, die auf den 32 Hauptkomponenten jugendlichen Medienhandelns mehr als zehn fehlende Werte aufwiesen. Cramers V = 0,33 ( $<<0,001)$.

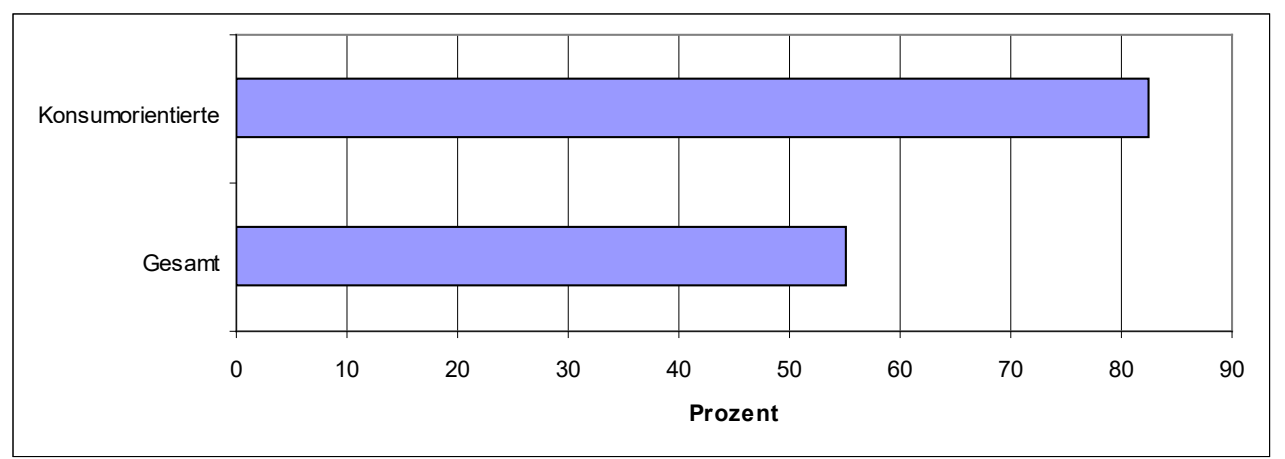

Abb. 4: Prozentualer Anteil von technikinteressierten Jugendlichen in der Gesamtstichprobe und im Cluster der Konsumorientierten (Für die Darstellung wurden die Kategorien „ziemlich" und „sehr" zusammengefasst; $n$ [Gesamt] = 1647; $n$ [Konsumorientierte] $=290$ ). Die Angaben zum Technikinteresse der Jugendlichen sind der Frage Nr. 25 des Fragebogens: „Interessierst du dich für Technik oder interessierst du dich nicht dafür?“ entnommen. Die Beantwortung der Frage erfolgte auf Grundlage einer vierstufigen Skala mit den kategorialen Ausprägungen (1) gar nicht, (2) wenig, (3) ziemlich und (4) sehr. Ausgeschlossen aus den Analysen wurden die Fälle, die auf den 32 Hauptkomponenten jugendlichen Medienhandelns mehr als zehn fehlende Werte aufwiesen. Cramers V = 0,28 (p<0,001).

lenklischees bestätigend - das ausgeprägte Technikinteresse der in diesem Cluster versammelten Jugendlichen (vgl. Abbildung 4): So geben 82 Prozent aller Konsumorientierten an, dass sie sich „ziemlich“ oder „sehr“ für technische Zusammenhänge interessieren. In der Untersuchungsstichprobe insgesamt beträgt der entsprechende Anteil nur rund 55 Prozent. 


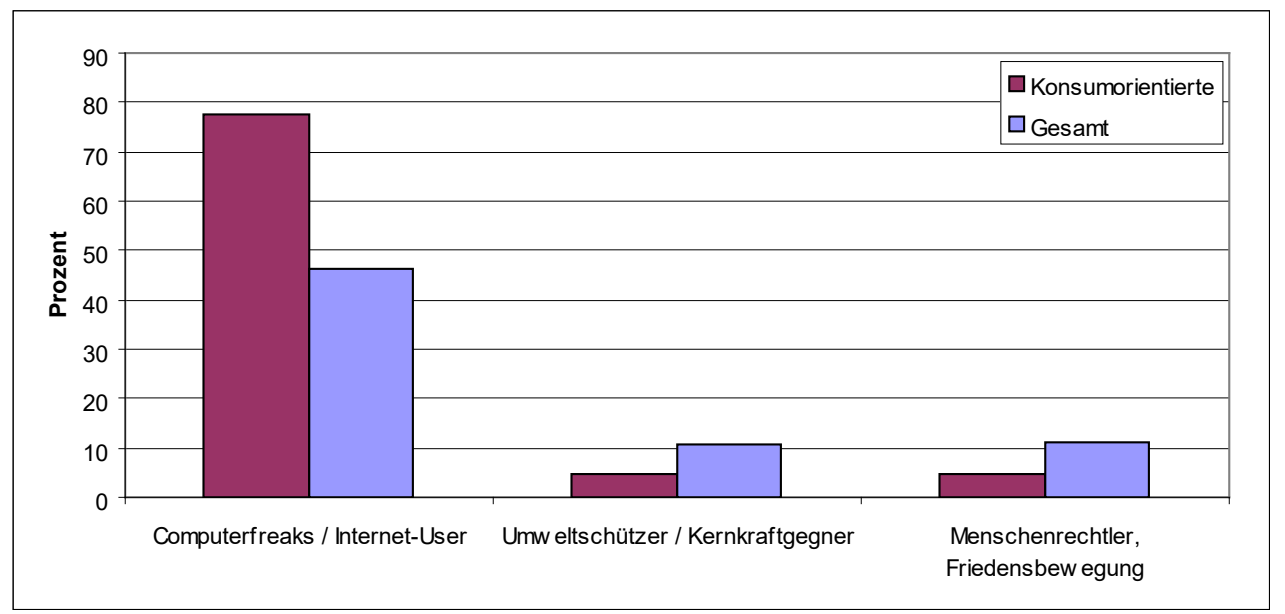

Abb. 5: Informelle Szenezugehörigkeiten in der Gesamtstichprobe und im Cluster der Konsumorientierten. $(n$ [Gesamt] $=1639$ bis 1641; $n$ [Konsumorientierte] = 288). Die Angaben zu den informellen Szenezugehörigkeiten sind der Frage Nr. 29 des Fragebogens: „Fühlst du dich einer der folgenden Gruppierungen zugehörig oder nicht zugehörig?“ entnommen. Die Beantwortung der Frage erfolgte durch einfaches Ankreuzen der in Frage stehenden informellen Szenen. Ausgeschlossen aus den Analysen wurden die Fälle, die auf den 32 Hauptkomponenten jugendlichen Medienhandelns mehr als zehn fehlende Werte aufwiesen. Computerfreaks/Internet-User: V = 0,29 ( $<<0,001)$; Umweltschützer/Kernkraftgegner: $\mathrm{V}=0,09$ ( $\mathrm{p}<0,001)$; Menschenrechtler/Friedensbewegung: $\mathrm{V}=0,10(\mathrm{p}<0,001)$.

Für die Beschreibung dieses Clusters scheinen die informellen Szenezugehörigkeiten von Bedeutung zu sein (vgl. Abbildung 5). In Einklang mit dem großen Interesse der Konsumorientierten für den Bereich Neuer Medien steht so etwa, dass Jugendliche, die sich der Szene der Computerfreaks und Internetuser zugehörig fühlen, in diesem Cluster mit etwa 78 Prozent - verglichen mit der Gesamtstichprobe (46,4 Prozent) - signifikant überrepräsentiert sind. Im Weiteren zeigen die Konsumorientierten eine verstärkte Distanz gegenüber solchen Jugendszenen, die sich gesellschaftspolitischen Zielsetzungen verpflichtet fühlen. So sind Jugendliche, die sich der Szene der Umweltschützer und Kernkraftgegner oder auch den Menschenrechtlern und Friedensbewegten verbunden fühlen, in diesem Cluster mit Anteilswerten von jeweils etwa 4,5 Prozent (Gesamtstichprobe: Umweltschützer und Kernkraftgegner: 10,8 Prozent; Menschenrechtler und Friedensbewegte: 11,1 Prozent) signifikant unterrepräsentiert.

Deutet man die subjektiven Szenezugehörigkeiten der Jugendlichen im Sinne dominierender sozialer Orientierungsmuster, so hat es den Anschein, als ob die Angehörigen dieses Clusters kommunitär-gemeinschaftlichen und gesellschaftspolitischen Belangen verglichen mit anderen Jugendlichen - eher distanziert gegenüberstehen. Die subjektive Zugehörigkeit zur Szene der Computerfreaks und Internetuser deutet im Weiteren darauf hin, dass das verstärkte Interesse an Neuen Medien bei den Angehörigen dieses Clusters auch mit Blick auf die Aufnahme von Peer-Kontakten von Relevanz zu sein scheint. 


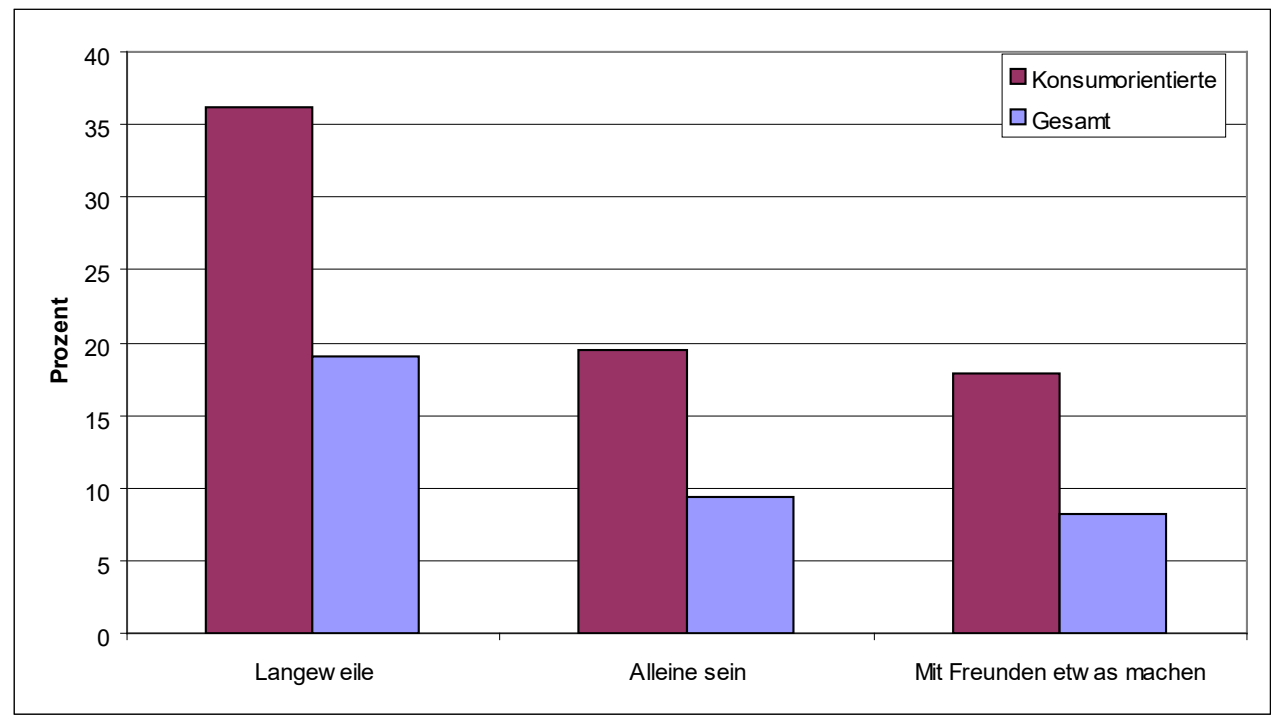

Abb. 6: Soziale und situative Kontexte der Computer- und Internetnutzung in der Gesamtstichprobe und im Cluster der Konsumorientierten (n [Gesamt] = 1545 bis 1623; $n$ [Konsumorientierte] $=265$ bis 283). Die Angaben zu den sozialen und situativen Kontexten der Computer- und Internetnutzung sind der Frage Nr. 33 des Fragebogens: „Welches Medium nutzt du bevorzugt in folgenden Situationen?“ entnommen. Als situative Kontexte der Mediennutzung wurden hier in der Form einzelner Frageitems genannt: „Wenn ich mich langweile“, „Wenn ich mich gezielt über etwas informieren will“, „Wenn ich abschalten will“, „Wenn ich allein sein möchte“ und „Wenn ich gemeinsam etwas mit Freunden machen möchte“. Die Beantwortung der Frage erfolgte auf Grundlage einer neunstufigen polytomen Skala mit den Ausprägungen: (1) „TV“, (2) „Radio“, (3) „Kino“, (4) „Video“, (5) „Computer/Internet“, (6) „Zeitung/Zeitschrift“, (7) „Comic“, (8) „Bücher“ und (9) „Keins davon“. Ausgeschlossen aus den Analysen wurden die Fälle, die auf den 32 Hauptkomponenten jugendlichen Medienhandelns mehr als zehn fehlende Werte aufwiesen. Langeweile: $V=0,23$ ( $p<0,001)$; Alleine sein: $V=0,19$ ( $p<0,001)$; Mit Freunden etwas machen: $\mathrm{V}=0,17(\mathrm{p}<0,001)$.

In Betrachtung der sozialen und situativen Kontexte der Mediennutzung bei den Konsumorientierten fällt im Weiteren auf, dass diese den Neuen Medien Computer und Internet in den verschiedenen Bereichen medienbezogener Aktivitäten übergreifend eine sehr viel höhere Nutzungspriorität einräumen als andere Jugendliche (vgl. Abbildung 6). Neue Medien haben für sie eine instrumentelle Funktion sowohl in Situationen, in denen sie allein sein möchten, als auch unter Umständen, in denen sie sich langweilen oder gemeinsam mit Freunden etwas unternehmen wollen. Offenbar haben die Konsumorientierten den Bereich der Neuen Medien nicht nur in ihren multifunktionalen Verwendungsmöglichkeiten für ihr individuelles Alltagshandeln erschlossen, sondern nutzen sie ebenfalls explizit für gemeinsame Aktivitäten mit Peers.

Schließlich zeigen die bivariaten Analysen, dass sich Konsumorientierte partiell auch in den Bereichen nicht-medienbezogener Freizeitaktivitäten von anderen Jugendlichen unterscheiden (vgl. Abbildung 7). So sind Jugendliche, die in ihrer Freizeit Zuhause „Hilfsdienste“ leisten resp. sich in Kirchen, Vereinen oder Parteien engagieren, unter den Angehörigen dieses Clusters signifikant unterrepräsentiert. Ebenfalls deutlich seltener als andere Jugendliche besuchen sie 


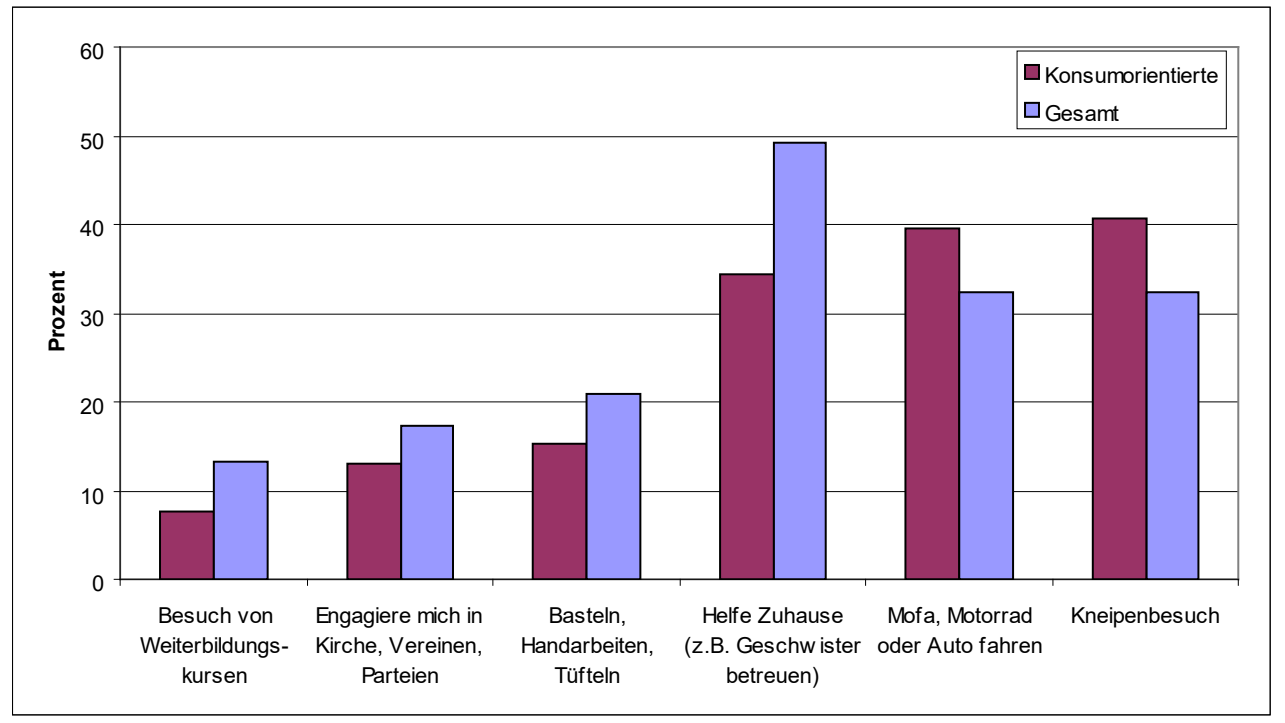

Abb. 7: Nicht medienbezogene Freizeitaktivitäten in der Gesamtstichprobe und im Cluster der Konsumorientierten (Für die Darstellung wurden die Kategorien „manchmal“ und „häufig “ zusammengefasst; $n$ [Gesamt] $=1658$ bis 1662; $n$ [Konsumorientierte] $=290$ ). Die Angaben zu den nicht medienbezogenen Freizeitaktivitäten der Jugendlichen sind der Frage Nr. 23 des Fragebogens: „Wie häufig beschäftigst du dich in deiner Freizeit mit den folgenden Dingen?“ entnommen. Den Jugendlichen wurde im Weiteren eine Anzahl verschiedener Freizeitaktivitäten genannt, mit der Bitte anzugeben, mit welcher Häufigkeit sie diesen Aktivitäten nachgehen. Die Beantwortung der Frage erfolgte über eine vierfach abgestufte Häufigkeitsskala mit den Ausprägungen (1) „nie“, (2) „selten“, (3) „manchmal“ und (4) „häufig“. Ausgeschlossen aus den Analysen wurden die Fälle, die auf den 32 Hauptkomponenten jugendlichen Medienhandelns mehr als zehn fehlende Werte aufwiesen. Besuch von WB-Kursen : V = 0,10 ( $\mathrm{p}=0,001)$; Engagement in Kirche, Vereinen, Parteien: V = 0,07 ( $\mathrm{p}=0,031)$; Basteln, Handarbeiten, Tüfteln: $\mathrm{V}=0,13(\mathrm{p}<0,001)$; Helfe Zuhause: $\mathrm{V}=0,17(\mathrm{p}<0,001)$; Mofa, Motorrad oder Autofahren: $\mathrm{V}=0,09(\mathrm{p}=0,005)$; Kneipenbesuch: $\mathrm{V}=0,10$ ( $\mathrm{p}=0,001)$.

in ihrer Freizeit außerschulische Weiterbildungskurse oder gehen kreativ-künstlerischen Aktivitäten nach (Basteln, Handarbeiten, Tüfteln). Demgegenüber treffen sie sich signifikant häufiger als andere mit ihren Freunden in Kneipen oder fahren mit dem eigenen Mofa, Motorrad oder Auto. Insgesamt hat es so den Anschein, als ob das Medienhandeln dieser Jugendlichen auch auf der Ebene dieser nicht-medienzentrierten Freizeitaktivitäten in einen stärker konsumtiv ausgerichteten Lebensstil eingebettet ist.

\subsection{Die mit Hilfe von qualitativen Methoden gewonnenen Portraits der Clustertypen -} am Beispiel der „Konsumorientierten“

Im Folgenden werden am Beispiel der interaktiven Mediennutzung einzelne Ergebnisse aus den Inhaltsanalysen der Interviews mit Jugendlichen aus dem Cluster der „Konsumorientierten“ vorgestellt, um das methodische Vorgehen nach dem Leitbild der Triangulation zu verdeutlichen. Zunächst wird jedoch 
der Ablauf der Datenerhebung und der Generierung der Interpretationen kurz dargestellt.

\subsubsection{Forschungsmethodischer Exkurs: Auswahl der Interviewfälle, Leitfadenkonstrukti-} on, qualitative Inhaltsanalyse

Um als Interviewpartner möglichst prototypische Fälle befragen zu können, wurden zunächst alle Personen eines jeden Clusters hinsichtlich ihrer Nähe zum Clusterschwerpunkt - gemessen über die Größe der quadrierten euklidischen Distanz zwischen dem jeweiligen Jugendlichen und dem Clustercentroid - einem Ranking unterzogen und anschließend versucht, möglichst rangniedrige Fälle zu einer Teilnahme zu motivieren. Da dieses methodische Vorgehen erfolgreich verlief, konnten jene Jugendlichen befragt werden, die sehr nahe am Clusterzentrum ${ }^{6}$ lagen. Alle Interviews wurden im Zeitraum von Juni bis Juli 2002 mit Hilfe eines Leitfadens von den Projektmitarbeitern durchgeführt. Bei der Konzeption des Leitfadens war darauf Wert gelegt worden, zum einen alle Dimensionen des Bielefelder Medienkompetenzmodells abzubilden und insbesondere Medienkritik zu thematisieren. Zum anderen sollten die Wahrnehmungen und Deutungen der Jugendlichen in ihren lebensweltlichen Kontexten und die ihnen zur Verfügung stehenden Handlungsmöglichkeiten hervortreten können. Alle Interviews wurden nach den von Rosenthal (1987) formulierten Transkriptionsregeln verschriftlicht und anschließend in einem ersten Auswertungsschritt in Fallbeschreibungen zusammengefasst. In einem zweiten Schritt wurden die Interviews des Clusters der „Konsumorientierten“ mit dem Programm MaxQDA (Kuckartz 1999) computergestützt einer qualitativen Inhaltsanalyse unterzogen, die als Ergebnis ein Portrait der jeweiligen Clustertypen jugendlichem Medienhandelns lieferte. Die qualitative Inhaltsanalyse der sechs Interviews des Clusters wurde nach Mayring durchgeführt (Mayring 1997, 2002) und die erstellten Interpretationen anschließend im gesamten Forschungsteam diskutiert, um zu möglichst deckungsgleichen Deutungen zu gelangen.

\subsubsection{Ergebnisse der qualitativen Analysen}

Die Ergebnisse der qualitativen Analysen zur interaktive Mediennutzung der Jugendlichen des Clusters der „Konsumorientierten“ können allein schon aus Platzgründen im Folgenden nur an Beispielen illustriert werden. Die „Konsumorientierten“ weisen aufgrund der quantitativen Auswertung beim Umgang mit Computer und Internet weit überdurchschnittliche Nutzungszeiten auf, die sie häufig mit dem Downloaden unterschiedlicher medialer Inhalte, dem Installie-

6 Im Cluster der „Konsumorientierten“ konnten so die Fälle der Ränge 1, 2, 3,6, 7 und 9 von den insgesamt 290 Personen des Clusters befragt werden. 
ren von Programmen und dem Aufenthalt im Internet verbringen. Hierbei sind ihre Nutzungszeiten noch höher als die des Clusters der „Allrounder“.

Die Analysen der qualitativen Interviews zeigen jedoch weitere Gründe auf, die zu derart langen Nutzungszeiten führen (und diese relativieren). Zum einen benutzen die „Konsumorientierten“ den Computer in besonderem Maße als „Multifunktionsanlage“, d.h. er dient als Musikanlage, Heimkino und Datenzentrale, in der sie ihre persönlichen Besitzstände (z.B. Lieder, Videos, DVDs) archivieren und zur Verfügung halten. Der Computer wird von vielen „Konsumorientierten“ für mehr als nur die „klassischen“ Funktionen der Textverarbeitung, Kommunikation, Spiele und Recherche genutzt: „PC, da mach ich eigentlich alles drüber bei mir im Zimmer, da hab ich meine Musikanlage daran gekoppelt, und (...) halt auch meine Spiele so“ (Johannes, 17 Jahre, 114-1157). Funktionen, die der Unterhaltung der Rezipienten dienen, wie etwa einen selbst kopierten Film zu sehen oder Musik zu hören, nehmen einen großen Raum im Medienhandeln der „Konsumorientierten“ ein. Jan nutzt, wie Felix, Sascha und Johannes auch, seinen PC als Musikanlage: „Ich hör mit dem Computer viel Musik. Weil ich hab alle meine CD‘s, die ich so hab, (...) in son MP3 Format" (Jan, 15 Jahre, 326-327) und jener Jugendliche beschreibt dies anschließend als die für ihn wichtigste Funktion am Rechner, denn er hat „so über tausend Lieder (...) drauf“ (Jan, 15 Jahre, 331) und durch den PC einen schnellen Zugriff auf sein Musikarchiv.

Der PC wird von den Jugendlichen zumeist sofort in Betrieb genommen, wenn sie von der Schule oder der Ausbildung nach Hause kommen [,,meistens (...) dann direkt an den Rechner“ (Felix, 17 Jahre, 109-110); „Als ich gestern zu Hause war, bin ich eigentlich direkt an den Computer gegangen“ (Boris, 19 Jahre, 12)] oder „läuft eh die ganze Zeit"“"ist eh immer an". Zum anderen ist der PC mit seinen unterschiedlichsten Möglichkeiten für diese Jugendlichen auch das Medium, welches sie am liebsten nutzen, um Zeitlücken zwischen Aktivitäten oder Terminen zu überbrücken, oder um sich die Langeweile zu vertreiben. So hebt der 17-jährige Felix als erstes bei der Beschreibung seines Interesses am Internet hervor, dass „man (...) ziemlich viele Sachen (hat), die man machen kann, wenn einem langweilig ist oder so was" (Felix, 17 Jahre, 830-831), und Tim ergänzt: „Es gibt ellenviele Seiten, wo man was finden kann“ (Tim, 13 Jahre, 324). Auch der 19-jährige Sascha surft zur Beschäftigung durchs WWW und nutzt nicht nur die Möglichkeiten zum Downloaden und zur Informationsrecherche, sondern sitzt regelmäßig am Computer, wenn er nach den Hausaufgaben „nicht mehr viel zu tun“ (Sascha, 19 Jahre, 106) hat und er sich weder mit den Freunden noch mit seiner Freundin treffen kann. Jan spielt zwar auch gerne Computerspiele, denn „,der Computer (...) macht auch Spaß, aber das (ist) mehr so zur Beschäftigung“ (Jan, 15 Jahre, 200). Der PC ist für viele „Konsumorientierte“ ein Medium, das sich für sie besser als andere Medien dazu eignet, „um den Tag vorüber gehen zu lassen“ (Felix, 17 Jahre, 748).

7 Angabe der Zeilennummern des transkribierten Interviews. 
4.4 Zur Triangulation der quantitativ und qualitativ gewonnenen Befunde: Konvergenz, Komplementarität oder was?

Zunächst einmal ist zu konstatieren, dass sich das Leitprinzip der Triangulation im Kontext der von uns dargestellten Studie sowie deren Ergebnissen erfolgreich bewährt hat. Die Kombination verschiedener Erkenntnisstrategien wurde der Mehrdimensionalität des Untersuchungsproblems nicht nur gerecht, sondern führte darüber hinaus zu Ergebnissen und Befunden, mit deren Hilfe es möglich ist, die unterschiedlichen Dimensionen von Medienkompetenz im Jugendalter differenziert zu entfalten. Im Gegensatz zu zahlreichen Jugendstudien, die versuchen, ein einheitliches Bild von Jugend zu zeichnen, konnte die vorgestellte Untersuchung deutlich machen, dass pauschale Aussagen über medienkompetentes Handeln auch bei Heranwachsenden zu einem verkürzten Blickwinkel auf das Phänomen führen. Wie die von uns durchgeführten clusteranalytischen Untersuchungen zeigen, scheint es vielmehr gewinnbringend, eine differenzierende Betrachtungsweise zu forcieren, die nachvollzieht, dass sich die Generation der heutigen Jugendlichen - bezogen auf die Struktur ihres Medienhandelns - durchaus nicht homogen, sondern heterogen darstellt. Die von uns vorgenommene Zuordnung von Jugendlichen zu verschiedenen Typen des Medienhandelns versucht, diesem Sachverhalt Rechnung zu tragen.

Die Architektur der vorgestellten Studie orientierte sich sowohl in theoretischer als auch in methodologischer Hinsicht am Leitprinzip der Triangulation. Unter Berücksichtigung der Theorien-Triangulation konnte die Ausprägung von Medienkompetenz im Jugendalter umfassend und vor allem mehrperspektivisch beleuchtet werden. Aus dem Uses-and-Gratifications-Ansatz abgeleitete Untersuchungsfragen machten deutlich, dass Jugendliche sich ihre mediale Umwelt aktiv aneignen und sich dabei gemäß ihrer Bedürfnisse und angestrebten Gratifikationen unterschiedlicher Medien bedienen. Auch diese, so der Befund unserer Studie, differieren nach unterschiedlichen Clustern. Die empirischen Analysen machten so etwa für das Cluster der Konsumorientierten deutlich, dass es bei dessen Angehörigen insbesondere das Unterhaltungsbedürfnis sowie konsumtive Interessen sind, die den Umgang mit Medien leiten. Diese spezifischen Interessen werden in jugendkulturellen Szenen weiter ausformuliert und unterstützt. $\mathrm{Zu}$ einer Aktivierung sozialen Kapitals, beispielsweise in Form von Unterstützung bei Problemen im Umgang mit den Neuen Medien, führt dieses - zumindest in der Gruppe der Konsumorientierten - allerdings nicht. Die Perspektive des Bourdieu'schen Kapitalsortenansatzes ermöglichte es in diesem Zusammenhang, entsprechende Erkenntnisse theoretisch einzuordnen und so zentrale Rahmenbedingungen des Erwerbs und der Ausübung von Medienkompetenz in den Blick zu nehmen. In das theoretische Konzept der Wissensklufthypothese schließlich lassen sich Untersuchungsergebnisse einordnen, die sich in der Dimension des Medienwissens ergeben. Dabei zeigt sich, dass Kompetenz in dieser Dimension keineswegs pauschal als Wissen oder Unwissen klassifiziert werden kann. So zeigen die Konsumorientierten ja ein eher 
durchschnittliches literarisches Bildungswissen, weisen aber ein extrem überdurchschnittlich ausgeprägtes Wissen über das Mediensystem auf. Die Ergebnisse machen deutlich, dass der von uns gewählte mehrperspektivische Ansatz zu differenzierten Aussagen führt, die sich gemäß der Theorien-Triangulation ergänzen und zu einem umfassenden Bild des Medienhandelns Jugendlicher führen.

Entsprechende Differenzierungen lassen sich auch durch die Methoden-Triangulation erzielen, die wir durch die Kombination qualitativer und quantitativer Verfahren erreichten. Dabei hat es sich unter den Prämissen unserer Studie (vgl. Abschnitt 3.2) bewährt, das „klassische“ Phasenmodell der Methodenkombination, gemäß dem zuerst qualitative Verfahren zur Hypothesenkonstruktion und danach quantitative Methoden zur Hypothesenüberprüfung eingesetzt werden, umzukehren. Nachdem zunächst durch eine repräsentative Erhebung Aussagen über Häufigkeitsverteilungen von Merkmalskonstellationen sowie vor allem über Zusammenhänge zwischen Variablen in der Grundgesamtheit der Jugendlichen gemacht werden konnten, gelang es uns anschließend, die befragten Jugendlichen in ihrem vielfältigen Medienhandeln auf einige wenige Cluster zu „verdichten“. Die Validität der clusteranalytischen Lösung konnte durch das Heranziehen externer Variablen belegt werden. So bestätigte sich beispielsweise im Cluster der Konsumorientierten die Annahme, dass eine Gruppierung Jugendlicher, die eine Nutzung insbesondere der Neuen Medien intensiv praktiziert, eine signifikant höheren Anteil männlicher und komplementär dazu einen geringeren Prozentsatz weiblicher Heranwachsender aufweist. Auch bestimmte informelle Szenezugehörigkeiten der befragten Jugendlichen fungierten als relevante Einflussgrößen. Bezogen auf das Cluster der Konsumorientierten bestätigte sich auch hier die Erwartung, dass sich die Angehörigen dieses Clusters überzufällig der Szene der Computerfreaks und Internetuser zuordnen und ein ausgeprägtes Technikinteresse aufweisen. Die sich anschließenden qualitativen Interviews konnten die erzielten Ergebnisse im Sinne des „Konvergenzmodells“ bzw. der „Integrationsthese“ bestätigen, so dass die Verlässlichkeit und Gültigkeit der Aussagen der quantitativen Befragung gestützt wurde. Die Anlage der qualitativen Untersuchung machte es aber darüber hinaus möglich, ergänzende Informationen zu sammeln, die nun im Sinne der „Komplementaritätsthese “ - zu breiteren, vielfältigeren und tieferen Erkenntnisse über das Medienhandeln Jugendlicher und die Ausprägung von Medienkompetenz führten. So zeigt sich im Cluster der Konsumorientierten, dass die unterhaltungsorientierte Nutzung der Neuen Medien nicht mit grundsätzlicher Passivität zu verbinden ist, sondern durchaus kreative Formen des Medienumgangs beinhaltet, wenn der PC als „Multifunktionsanlage“ genutzt wird. Auch wird deutlich, dass Mediennutzung trotz Konsumorientierung nicht zum allumfassenden Lebensinhalt wird, der andere Interessen nachhaltig in den Hintergrund treten lässt. Ist der PC zwar einerseits ein beliebtes Unterhaltungsmedium der Konsumorientierten, nutzen sie diesen andererseits aber vornehmlich dann, wenn Zeitlücken zu überbrücken sind. Die Erfassung des sozialen Kontextes sowie 
der Deutungen und Pläne der in ihm handelnden Subjekte erlaubt es somit, die Einbettung von Medien und deren Nutzung in die Lebenswelten und Biographien von Jugendlichen zu rekonstruieren. Die dabei entstehenden Deutungsmuster und Handlungsperspektiven ermöglichen ein umfassendes Bild jugendlichen Medienhandeln, das die Aussagen quantitativer Daten bestätigt und ergänzt. Am Beispiel des Clusters der Konsumorientierten haben wir deutlich gemacht, dass das Leitprinzip der Triangulation sowohl im Sinne des Konvergenzmodells als auch im Sinne der Komplementaritätsthese - verstanden als Methoden-Triangulation - zu umfassenderen und weitreichenderen Forschungsergebnissen führt, als es bei der Anwendung nur einer Methodenart der Fall wäre, um Profile von Medienhandeln im Jugendalter differenziert zu beschreiben. Der Erkenntnisgewinn ist dabei keine Frage der isolierten Anwendung quantitativer oder qualitativer Forschungsmethoden, sondern ergibt sich gerade aus deren Kombination.

\section{Literatur}

Abel, J./Möller, R./Treumann, K P: Einführung in die Empirische Pädagogik. Stuttgart 1998. Arabie, P./ Hubert, L.J./de Soete, G.: Clustering and Classification. Singapore u.a. 1996.

Baacke, D.: Medienkompetenz als Netzwerk. Reichweite und Fokussierung eines Begriffs, der Konjunktur hat. In: Medien praktisch, Heft 2, 1996a, S. 4-10.

Baacke, D.: Medienkompetenz - Begrifflichkeit und sozialer Wandel. In: von Rein, A. (Hrsg.): Medienkompetenz als Schlüsselbegriff. Bad Heilbrunn 1996b.

Baacke, D./Sander, U./Vollbrecht, R.: Lebenswelten sind Medienwelten. Opladen 1990.

Baacke, D./Sander, U./Vollbrecht, R./Kommer, S.: Zielgruppe Kind. Kindliche Lebenswelten und Werbeinszenierungen. Opladen 1999.

Bacher, J.: Clusteranalyse. Anwendungsorientierte Einführung. 2. Aufl. München/Wien 1996.

Bacher, J.: P 36, P 37 Clusteranalyse. Handbuch zum Almo Statistik System. Linz 2002.

Backhaus, K u.a.: Multivariate Analysemethoden. 8. Aufl. Berlin u.a. 1998.

Bailey, K D.: Typologies and Taxonomies. An Introduction to Classification Techniques. Thousand Oaks u.a. 1994.

Bortz, J.: Statistik für Sozialwissenschaftler. 5. Aufl. Berlin u.a. 1999.

Denzin, N. K: The Research Act: A Theoretical Introduction to Sociological Methods. 3. Aufl. New York 1989. (1. Aufl. 1970).

Eckes, T./Rossbach, H.: Clusteranalysen. Stuttgart u.a. 1980.

Engler, S.: Zur Kombination von qualitativen und quantitativen Methoden. In: Friebertshäuser, B./ Prengel, A. (Hrsg.): Handbuch Qualitativer Forschungsmethoden in der Erziehungswissenschaft. Weinheim/München 1997, S. 119-130.

Everitt, B. S.: Cluster Analysis. 3. Aufl. London u.a. 1993.

Flick, U: Qualitative Forschung. Theorie, Methoden, Anwendung in Psychologie und Sozialwissenschaften. Reinbek bei Hamburg 1995.

Flick, U. u.a. (Hrsg.): Handbuch qualitativer Sozialforschung. Grundlagen, Konzepte, Methoden und Anwendungen. München 1991.

Gordon, A. D.: Classification. 2. Aufl. Boca Raton (Florida) u.a. 1999.

Kelle, U./Erzberger, C.: Integration qualitativer und quantitativer Methoden. Methodologische

Modelle und ihre Bedeutung für die Forschungspraxis. In. Kölner Zeitschrift für Soziologie und Sozialpsychologie, Jg. 51, 1999, Heft 3, S. 509-531.

Kuckartz, U.: Computergestützte Analyse qualitativer Daten. Opladen u. Wiesbaden 1999.

Mayring, P: Qualitative Inhaltsanalyse. Grundlagen und Techniken. 6. Aufl. Weinheim 1997. Mayring, P: Einführung in die qualitative Sozialforschung. 5. Aufl. Weinheim 2002. 
Rosenthal, G.: „... wenn alles in Scherben fällt ...“. Von Leben und Sinnwelt der Kriegsgeneration. Typen biographischer Wandlungen. Opladen 1987.

Saldern, M. von: Zum Verhältnis von qualitativen und quantitativen Methoden. In: König, E./Zedier, P. (Hrsg.): Bilanz qualitativer Forschung. Band 1: Grundlagen qualitativer Forschung. Weinheim 1995, S. 331-371.

Tabachnick, B. G./Fidell, L. S.: Using Multivariate Statistics. 3. Aufl. New York 1996.

Treumann, K P: Zum Verhältnis qualitativer und quantitativer Forschung. In: Heitmeyer, W. (Hrsg.): Interdisziplinäre Jugendforschung. Fragestellungen, Problemlagen, Neuorientierungen. Weinheim 1986.

Treumann, K P: Triangulation als Kombination qualitativer und quantitativer Forschung. In: Abel, J./ Möller, R./Treumann, K P: Einführung in die Empirische Pädagogik. Stuttgart 1998, S. 154-182.

Treumann, K P./Burkatzki, E./Strotmann, M./Wegener, C.: Hauptkomponentenanalytische Untersuchungen zum Medienhandeln Jugendlicher: In: Bachmair, B./Diepold, P./de Witt, C.: Jahrbuch Medienpädagogik 4. Wiesbaden 2004, S. 145-168. http://dx.doi.org/10.21240/mpaed/retro/2017.08.09.X.

Webb, E. u.a.: Unobtrusive Measures. Chicago 1966.

Wolf, W: Qualitative versus quantitative Forschung. In: König, E./Zedier, P (Hrsg.): Bilanz qualitativer Forschung. Bd. I: Grundlagen qualitativer Forschung. Weinheim 1995, S. 309-329. 